\title{
Relationship between childhood adversity and bipolar affective disorder: systematic review and meta-analysis
}

\author{
J. E. Palmier-Claus, K. Berry, S. Bucci, W. Mansell and F. Varese
}

\section{Background}

The relationship between childhood adversity and bipolar affective disorder remains unclear.

\section{Aims \\ To understand the size and significance of this effect through a statistical synthesis of reported research.}

\section{Method}

Search terms relating to childhood adversity and bipolar disorder were entered into Medline, EMBASE, PsycINFO and Web of Science. Eligible studies included a sample diagnosed with bipolar disorder, a comparison sample and a quantitative measure of childhood adversity.

\section{Results}

In 19 eligible studies childhood adversity was 2.63 times (95\% $\mathrm{Cl}$ 2.00-3.47) more likely to have occurred in bipolar disorder compared with non-clinical controls. The effect of emotional abuse was particularly robust (OR=4.04, 95\% Cl 3.12-5.22), but rates of adversity were similar to those in psychiatric controls.

\section{Conclusions}

Childhood adversity is associated with bipolar disorder, which has implications for the treatment of this clinical group. Further prospective research could clarify temporal causality and explanatory mechanisms.

\section{Declaration of interest}

None.

\section{Copyright and usage}

(c) The Royal College of Psychiatrists 2016.
Bipolar affective disorder is characterised by extreme depressive and manic affective states, which are often associated with adverse outcomes, including reduced functioning, impaired quality of life and increased risk of death by suicide. ${ }^{1-3}$ Response to treatment is limited, with high rates of relapse. ${ }^{4}$ A better understanding of the risk factors for bipolar disorder is vital for refining detection and intervention strategies. Although research has typically focused on the biogenetic determinants of bipolar symptoms, environmental risk factors are increasingly being considered. ${ }^{5}$ This review and meta-analysis explore the association between bipolar disorder and childhood adversity.

Childhood adversity is associated with a variety of negative outcomes in the general population. ${ }^{6}$ In individuals with bipolar affective disorder it has been linked to increased mood cycling, greater numbers of affective episodes and the presence of psychosis. ${ }^{7,8}$ However, the question of whether childhood adversity relates to the development of this disorder remains unresolved. Previous reviews have observed high rates of adversity in many, but not all, samples of people with bipolar disorder. ${ }^{9-14}$ To date, no research has attempted to integrate empirical findings using meta-analytic methods. To do so would provide a more rigorous method for testing the null hypothesis, and also allow for consideration of the size and consistency of the effects.

Authors have proposed that emotional abuse and neglect may convey greater risk of bipolar disorder than other forms of maltreatment such as sexual or physical abuse. ${ }^{5}$ Comparison of effect sizes for different forms of adversity might help to clarify whether specific adversity subtypes are more strongly related to bipolar symptoms. Meta-analytic approaches might also elucidate whether childhood adversity is associated with a particular form of bipolar disorder. Type 1 bipolar disorder is characterised by periods of mania (episodes of extremely elated mood, arousal and levels of activity, often in the presence of psychosis), whereas type 2 disorder presents only attenuated symptoms of mania with limited impact on functioning (hypomania). Given the evidence for an association between adversity and severe psychopathologic disorder, characterised by psychotic symptoms, ${ }^{15}$ levels of childhood adversity may be elevated in patients with type 1 disorder. Lastly, diagnoses of major depression and schizophrenia appear more likely in individuals with a history of childhood adversity. ${ }^{15,16}$ It is possible that childhood maltreatment is related to one particular form of psychiatric disorder. The final and exploratory aim of this review was therefore to compare rates of childhood adversity in individuals diagnosed with bipolar disorder with those in people diagnosed with schizophrenia or major depression.

We examined three a priori hypotheses: first, rates of childhood adversity would be elevated in samples with bipolar disorder compared with non-clinical controls; second, effect sizes for emotional abuse and neglect would be higher than for other forms of adversity; and third, rates of childhood adversity would be greater in individuals with type 1 disorder compared with type 2. We made no prediction regarding rates of childhood adversity in bipolar disorder compared with the other clinical samples.

\section{Method}

The review was carried out in accordance with the Preferred Reporting Items for Systematic Reviews and Meta-Analyses (PRISMA) standards. A systematic search of four databases (Medline, EMBASE, PsycINFO and Web of Science) identified peer-reviewed articles published between January 1980 and October 2014. We used blocks of search terms pertaining to bipolar disorder (bipolar, mani*, cyclothymi ${ }^{\star}$, manic-depressi ${ }^{\star}$ OR hypomani*) AND childhood adversity (child abuse, physical abuse, sexual abuse, psychological abuse, emotional abuse, neglect $^{*}$, trauma ${ }^{*}$, advers ${ }^{*}$, maltreat ${ }^{\star}$, bully ${ }^{*}$, bullied, victim ${ }^{*}$ OR parental loss). The search terms were partly adapted from past reviews, ${ }^{10,11,15}$ and where possible were 'exploded' in the field of Bipolar Disorder. The search in Web of Science was restricted to the areas of psychiatry and psychology by field. In addition to the systematic search, we screened the reference lists of the included manuscripts and previous reviews. ${ }^{5,9-14,17}$ We also examined 
journal articles citing at least one of the included studies. Where data were insufficient to assess eligibility or calculate an effect size, further information was requested from the study's corresponding author.

\section{Eligibility criteria}

The review included case-control studies (comparing two existing groups distinguished by a defining outcome, i.e. bipolar status $v$. control) and epidemiological (prospective and cross-sectional) studies, where a quantitative measure of childhood adversity was administered to individuals with a formal diagnosis of bipolar disorder according to the DSM (DSM-III or later) or ICD (ICD-9 or -10). We defined childhood adversity as the experience of neglect, abuse, bullying or the loss of parents before the age of 19 years. Studies exploring loss through separation (e.g. divorce of parents), expressed emotion and/or stressful life events occurring in adulthood (after the age of 18 years) were not included. We excluded relatively common parenting practices (e.g. spanking, shouting), as these were assumed to be subject to cultural variability. Also excluded were case-note reviews that opportunistically assessed - rather than systematically measured - childhood adversity, owing to the increased likelihood of response bias. When both 12-month and lifetime diagnoses were provided, the latter were selected for effect size extraction. ${ }^{18}$ Only articles published in peer-reviewed English-language journals were included in the analysis.

Only studies with at least one eligible control sample were included. These samples were defined a priori as comprising healthy individuals without an identified DSM or ICD diagnosis (in the epidemiological studies, this was defined as respondents known to be free of the outcome of interest, i.e. bipolar disorder), and individuals with a DSM or ICD diagnosis of major depression or non-affective psychosis (e.g. schizophrenia, schizoaffective disorder, schizophreniform disorder or delusional disorder).

\section{Screening and data extraction}

The lead author (J.P.C.) screened articles in three stages: at title level, abstract level and article level. A third of titles (1800) were double-rated separately by a postgraduate researcher, with adequate levels of agreement $(95 \%, \kappa=0.65)$. All of the abstracts (446) were double-rated with similarly high levels of agreement $(87 \%, \kappa=0.71)$. The majority of discrepancies were due to the primary coder (J.P.C.) being overly inclusive. Two authors extracted data and calculated effect sizes using a data spreadsheet. The intraclass correlation (ICC) between the two sets of effect sizes indicated high levels of agreement $(\mathrm{ICC}=0.98, P<0.001)$. For the four cases where the primary authors were in disagreement, the wider team arbitrated. Extracted data included study and effect size descriptors. When possible the authors extracted binary (e.g. frequency tables, percentages), as opposed to $d$-family (e.g. means, standard deviations), effect sizes based on the use of odds ratio as the overall metric.

\section{Methodological quality}

Methodological quality was explored using the Newcastle-Ottawa Quality Assessment Scale (NOQAS), ${ }^{19}$ which assesses the selection and comparability of the samples and the suitability of the adversity exposures. Gender was selected as the most important covariate or matching criterion, given the studies showing greater levels and impact of childhood adversity in women compared with men. ${ }^{20}$ Quality ratings were based on the effect sizes of interest, rather than other analyses reported in the papers. Independent, masked quality ratings by a postgraduate researcher demonstrated good interrater reliability with the lead author (ICC $=0.83$, $P<0.001)$.

\section{Statistical analysis}

We used Comprehensive Meta-Analysis (version two) to compute effect sizes and conduct the analyses. All effect sizes were converted to odds ratios to aid interpretation of the results. Effects were integrated using random effects meta-analysis. Visual inspection of funnel plots and regression tests of funnel plot asymmetry (Egger's test) established the presence of publication and selection bias. Where selection bias was deemed likely, Duval \& Tweedie's trim and fill method was employed to identify and correct for hypothetically missing effects.

The analysis consisted of four stages. In stage one we considered the overall effects from studies comparing bipolar and non-clinical samples on measures of childhood adversity. This analysis focused on the association between childhood adversity and bipolar disorder regardless of adversity type, and considered both single (e.g. sexual abuse) and multiple (e.g. sexual and emotional abuse) exposures. When extracting data in the presence of more than one measure of adversity we used the most global or wide-reaching assessment (e.g. total levels of adversity). Where this information was unavailable, we contacted the corresponding author of the primary manuscript to request information regarding an aggregated effect. In the absence of this information, we calculated separate effect sizes for each type of adversity, which were then aggregated in the main analysis. The second stage of analysis examined independent associations between different types of exposures and bipolar disorder. In the third stage overall effects were extracted for studies that compared childhood adversity between samples with type 1 and type 2 bipolar disorder. Finally, we independently examined differences in childhood adversity between bipolar disorder and other psychiatric groups (major depression, schizophrenia).

Some manuscripts contained the results of both the unadjusted analyses and those adjusting for covariates. In order to increase comparability among the eligible studies we included the unadjusted results in the main analyses and then conducted a sensitivity analysis with the adjusted effects. In the presence of multiple levels of adjustment we included the analysis with the largest number of demographic and/or clinical covariates. The majority of the aforementioned analyses explored the impact of childhood adversity generally, rather than the specific effects of adversity subtypes over and above the other forms of adversity. Therefore, we did not include effects that examined the impact of exposures while controlling for other types of childhood adversity (e.g. Stikkelbroek et al). ${ }^{21}$ The full review protocol (CRD42015017201) is available through the PROSPERO website (http://www.crd.york.ac.uk/PROSPERO).

\section{Results}

The screening procedure is summarised in Fig. 1 and the characteristics of the included articles are given in online Table DS1. ${ }^{18,21-26,28-48}$ Eleven authors provided clarification or further information from which to generate an effect size. Only 11 studies reported the exact prevalence of childhood adversity within bipolar samples, which ranged from $8 \%$ (Laursen et al) to $77 \%$ (Fowke et al), with a weighted average exposure of $10.5 \% .^{22,23}$ This estimate includes parental loss (4 studies), sexual abuse (3 studies) and composite adversity measures (4 studies). Thirteen case-control and six epidemiological studies were included in the main analysis. The case-control studies included 1259 cases and 1118 controls, whereas the epidemiological studies surveyed over 2.1 million respondents. The epidemiological research included three population-based cross-sectional design studies, ${ }^{21,24,25}$ two retrospective cohort design studies, ${ }^{18,22}$ and one quasi-prospective study. ${ }^{26}$ The latter examined childhood 


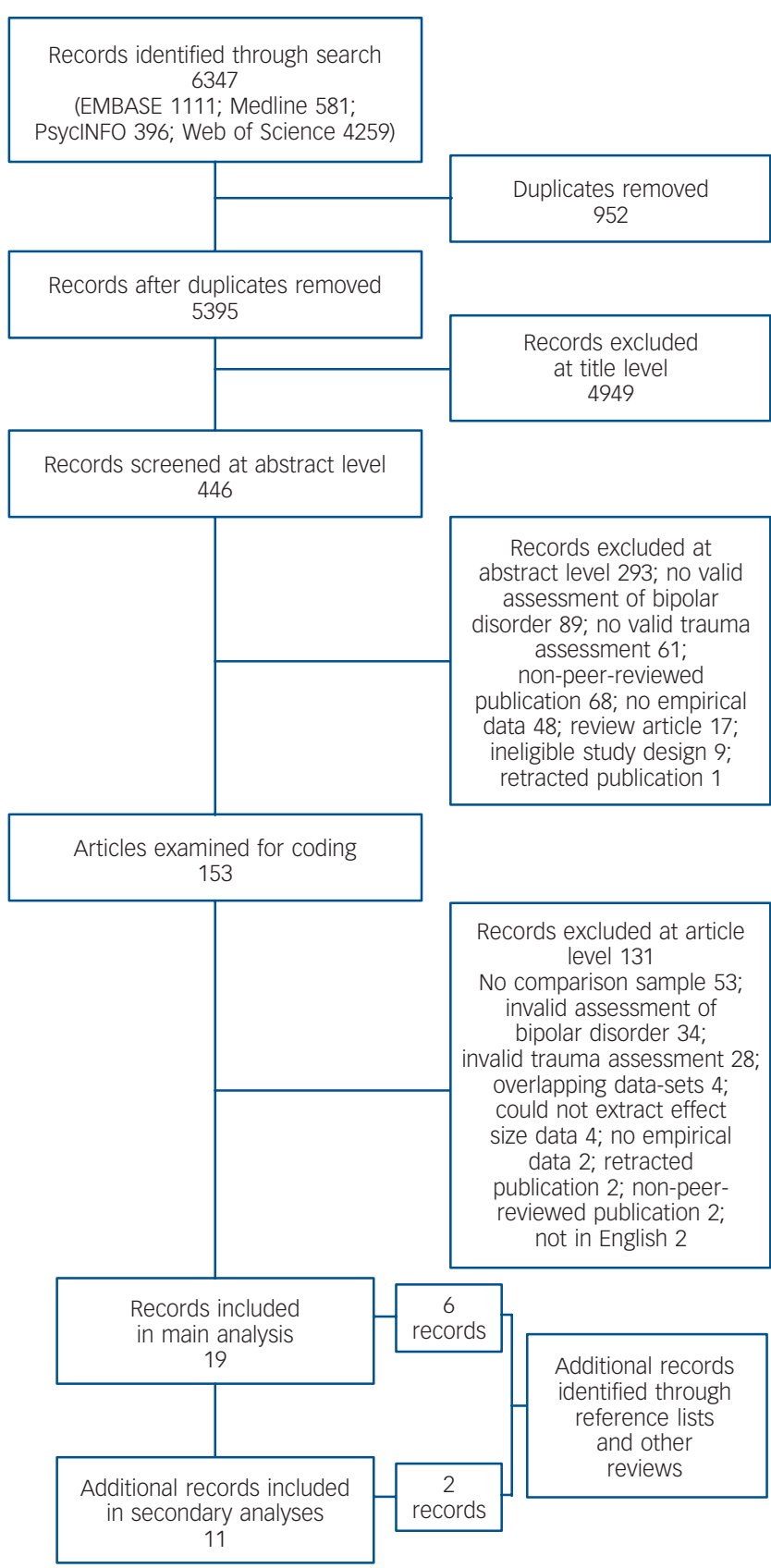

Fig. 1 Flow chart of literature screening

adversity as a predictor of transition to psychosis over a 3-year period in adulthood. The cohort design studies linked data on current diagnosis to registers on parental loss and child protection status. ${ }^{18,22}$ The most commonly used assessment of adversity in the case-control studies (seven studies) was the Childhood Trauma Questionnaire, a 28-item self-report measure of emotional and physical abuse, emotional and physical neglect and sexual abuse. ${ }^{27}$ Measures of childhood adversity in the epidemiological studies were generally single items derived from validated measures.

\section{Stage one}

Stage one investigated the overall association between childhood adversity and bipolar disorder. Figure 2 shows the ORs for each of the included studies, and the aggregated effects of childhood adversity on bipolar disorder. The analysis showed an overall effect of 2.63 (95\% CI $2.00-3.47, P<0.001$ ), suggesting that individuals with bipolar disorder are 2.6 times more likely to have experienced childhood adversity when compared with a non-clinical control group. Similar effect sizes were observed for the case-control ( $\mathrm{OR}=2.88,95 \%$ CI 2.04-4.06, $P<0.001)$ and epidemiological studies $(\mathrm{OR}=2.24,95 \% \mathrm{CI} 1.40-3.57, P=0.001)$. There was no significant difference $(Q(1)=0.74, P=0.391)$ in the strength of the effect sizes between the two subgroups.

\section{Heterogeneity analyses}

Heterogeneity was examined using the Q-test and $I^{2}$ statistics. Results showed that the strength of the relationship between childhood adversity and bipolar disorder varied considerably across studies $(Q(18)=79.53, \quad P<0.001)$, with $77 \%$ of the observed dispersion attributable to true statistical heterogeneity. This level of heterogeneity is generally thought to be high and should be considered when interpreting the results.

\section{Selection bias analyses}

Regarding publication bias, funnel plots of standard error against $\log$ odds ratios indicated a roughly symmetrical distribution of studies around the mean effect sizes. When combining the casecontrol and epidemiological literature the result of Egger's test was non-significant $(\beta=0.12$, s.e. $=1.08, P=0.456)$ indicating no evidence of publication or selection bias. Duval \& Tweedie's trim and fill found two hypothetical missing studies, which brought the imputed OR to 2.47 (95\% CI 1.8-3.1).

\section{Sensitivity analysis}

One-study-removed analysis suggested that the withdrawal of any particular study would not greatly alter the results. Three of the epidemiological studies provided effect sizes adjusted for covariates in addition to unadjusted scores. Repeating the analysis using adjusted scores yielded similar results $(\mathrm{OR}=2.58,95 \% \mathrm{CI}$ 1.96-3.36, $P<0.001)$ with equivalent levels of statistical heterogeneity $\left(Q(18)=79.2, P<0.001, I^{2}=77.27\right)$. This was also true when including only the epidemiological studies in the analysis $(\mathrm{OR}=2.14,95 \%$ CI $1.36-3.39, P=0.001)$.

\section{Stage two}

In stage two we examined associations between specific adversity subtypes and bipolar disorder. Table 1 shows the results of the analyses exploring whether specific types of childhood adversity are elevated in bipolar disorder. Grandin et al and Neeren et al both reported analyses from the Longitudinal Investigation of Bipolar Spectrum Disorders project; ${ }^{31,49}$ we selected information from the paper by Neeren et al because it specifically reported effects pertaining to the impact of adversity subtypes. The results of these separate meta-analyses showed significant effects of all childhood adversity subtypes, with the exception of parental loss, on bipolar disorder. Emotional abuse showed the strongest effect $(\mathrm{OR}=4.04,95 \%$ CI 3.12-5.22, $\mathrm{P}<0.001)$.

\section{Stage three}

Differences between bipolar disorder subtypes were studied in stage three. Four identified studies provided data to compare rates of childhood adversity across subtypes. ${ }^{32,38,39,47}$ No significant difference in childhood adversity was observed between type 1 and type 2 bipolar disorder $(\mathrm{OR}=0.93,95 \%$ CI $0.48-1.81$, $\left.P=0.827 ; Q(3)=6.91, P=0.075, I^{2}=56.58\right)$.

\section{Stage four}

In stage four we looked at differences between bipolar disorder and psychiatric controls, major depression and schizophrenia. Data from 11 studies were used to compare rates of childhood adversity in 
Table 1 Trauma subtype analyses

\begin{tabular}{|c|c|c|c|c|c|c|}
\hline & \multirow[b]{2}{*}{$k$} & \multicolumn{2}{|c|}{ Odds ratios } & \multicolumn{3}{|c|}{ Heterogeneity tests } \\
\hline & & OR $(95 \% \mathrm{Cl})$ & $P$ & $1^{2}$ & $Q$ & $P$ \\
\hline Physical abuse & 12 & $2.86(2.22-3.69)$ & $<0.001$ & 70 & 36.55 & $<0.001$ \\
\hline Sexual abuse & 12 & $2.58(2.08-3.20)$ & $<0.001$ & 35 & 16.94 & 0.109 \\
\hline Emotional abuse & 9 & $4.04(3.12-5.22)$ & $<0.001$ & 23 & 10.40 & 0.238 \\
\hline Physical neglect & 7 & $2.26(1.74-2.93)$ & $<0.001$ & 0 & 5.41 & 0.492 \\
\hline Emotional neglect & 7 & $2.62(2.03-3.38)$ & $<0.001$ & 0 & 5.94 & 0.430 \\
\hline Parental loss & 5 & $1.16(0.75-1.78)$ & 0.514 & 51 & 8.23 & 0.084 \\
\hline
\end{tabular}

bipolar disorder and major depression (see online Fig. DS1). Childhood adversity was significantly greater in bipolar disorder (OR $=1.24,95 \%$ CI 1.02-1.50, $P=0.031$ ), with low levels of statistical heterogeneity $\left(Q(10)=12.83, P=0.233, I^{2}=22.08\right)$. However, Egger's test approached significance $(\beta=0.75$, s.e. $=0.43, P=0.058)$, indicating the possibility of publication bias. After Duval \& Tweedie's trim and fill adjusted for three hypothetical missing studies, the imputed OR fell to 1.09 (95\% CI 0.88-1.36). Based on the post hoc hypothesis that the absence of an effect was due to the type of adversity considered, we repeated the analyses removing four studies that focused on parental loss. This elevated the effect size $\quad(\mathrm{OR}=1.54, \quad 95 \% \quad \mathrm{CI} 1.19-2.00, \quad P<0.001 ; \quad Q(6)=4.30$, $\left.P<0.001, I^{2}=0\right)$ showing significantly higher rates of childhood adversity in bipolar disorder when compared with major depression. No hypothetically missing study was detected, with no indication of publication bias $(\beta=-1.34$, s.e. $=1.25, P=0.166)$.

No significant difference in rates of childhood adversity was found when comparing bipolar disorder and schizophrenia in the analysis of five studies ( $\mathrm{OR}=0.89,95 \%$ CI $0.79-1.01$, $P=0.067 ; Q(4)=2.32, P=0.677, I^{2}=0$; see online Fig. DS2). Egger's test was non-significant $(\beta=-0.52, \quad$ s.e. $=0.42$, $P=0.152)$ and no hypothetically missing studies were estimated.

\section{Quality assessment}

The NOQAS ratings for the case-control studies are given in online Table DS2. Generally, the quality of the studies in the main analysis was adequate, with eight studies employing an appropriately matched control group and/or controlling for covariates in the analysis. Only one study failed to substantiate participants' diagnoses through interview. ${ }^{23}$ There was a nonsignificant trend of better study quality producing larger effects $(b=0.22$, s.e. $=0.12,95 \% \mathrm{CI}-0.01$ to $0.45 ; Z=1.82, P=0.066)$ in the case-control studies. Quality ratings for the case-control studies included in the secondary analysis were lower than for those in the main analysis. This was largely due to studies not controlling for covariates or employing matching criteria. The majority of the studies included in the secondary analysis employed a rigorous method of ascertaining diagnoses. Epidemiological studies included nationally representative samples with data obtained through structured interviews or record linkage; these studies adequately controlled for a range of covariates in their analyses, including gender.

\section{Discussion}

The results of the meta-analysis suggest that individuals with bipolar disorder are 2.6 times more likely to experience childhood adversity when compared with a non-clinical control group. This effect did not appear to be the result of study design or bias, and remained robust and significant even after controlling for hypothetically missing studies. The findings should be interpreted in the context of relatively few longitudinal studies and none with a
Study

Design

Adversity

Aas et al (2014) 36

Agid et al (1999) ${ }^{28}$

Chen et al (2014) $)^{37}$

Etain et al (2010) ${ }^{33}$

Fowke et al (2012) ${ }^{23}$

Furukawa et al (1999) ${ }^{29}$

Grandin et al (2007) ${ }^{31}$

Horesh \& lancu (2010) ${ }^{34}$

Janiri et al (2015) $)^{39}$

Konradt et al (2013) ${ }^{35}$

Rucklidge et al (2006) ${ }^{30}$

Savitz et al (2008) ${ }^{32}$

Watson et al (2014)

Subtotal

Afifi et al (2014) ${ }^{24}$

Gilman et al $(2014)^{26}$

Laursen et al (2007) ${ }^{22}$

Molnar et al (2001) 25

Scott et al (2010) ${ }^{18}$

Stikkelbroek et al (2012) ${ }^{21}$ Epidemiological Parental loss

subtotal

Total

$\begin{array}{llrrrr}\text { Case control } & \text { Trauma total } & 5.58 & 1.67 & 18.69 & 0.01 \\ \text { Case control } & \text { Parental loss } & 2.65 & 0.89 & 7.92 & 0.08 \\ \text { Case control } & \text { Physical abuse } & 1.92 & 1.39 & 2.64 & 0.00 \\ \text { Case control } & \text { Trauma total } & 2.94 & 1.87 & 4.62 & 0.00 \\ \text { Case control } & \text { Trauma total } & 5.81 & 2.37 & 14.28 & 0.00 \\ \text { Case control } & \text { Parental loss } & 0.68 & 0.30 & 1.53 & 0.35 \\ \text { Case control } & \text { Maltreatment } & 2.53 & 1.68 & 3.82 & 0.00 \\ \text { Case control } & \text { Parental death } & 0.96 & 0.44 & 2.13 & 0.93 \\ \text { Case control } & \text { Trauma total } & 3.60 & 2.16 & 5.99 & 0.00 \\ \text { Case control } & \text { Trauma total } & 7.73 & 4.04 & 14.78 & 0.00 \\ \text { Case control } & \text { Trauma total } & 1.68 & 0.22 & 12.80 & 0.62 \\ \text { Case control } & \text { Trauma total } & 4.47 & 2.28 & 8.77 & 0.00 \\ \text { Case control } & \text { Trauma total } & 4.82 & 2.17 & 10.69 & 0.00 \\ & & 2.88 & 2.04 & 4.06 & 0.00 \\ \text { Epidemiological } & \text { Trauma total } & 4.40 & 3.29 & 5.88 & 0.00 \\ \text { Epidemiological } & \text { Trauma total } & 2.74 & 2.08 & 3.61 & 0.00 \\ \text { Epidemiological } & \text { Parental loss } & 1.57 & 1.16 & 2.12 & 0.00 \\ \text { Epidemiological } & \text { Sexual abuse } & 4.37 & 1.70 & 11.22 & 0.00 \\ \text { Epidemiological } & \text { Maltreatment } & 1.86 & 1.00 & 3.46 & 0.05 \\ \text { Epidemiological } & \text { Parental loss } & 0.77 & 0.38 & 1.57 & 0.47 \\ & & 2.24 & 1.40 & 3.57 & 0.00 \\ & & 2.63 & 2.00 & 3.47 & 0.00\end{array}$

Statistics for each study

Odds ratio $(95 \% \mathrm{Cl})$

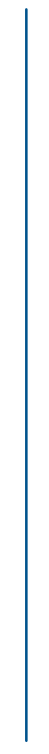

0.01

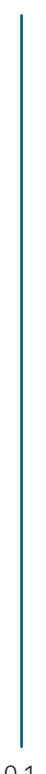

0.1

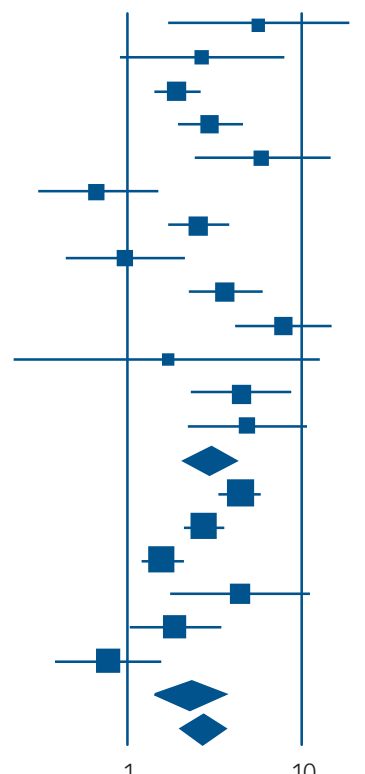

10

Fig. 2 Forest plot of effect sizes 
prospective cohort design, limiting the ability to make causal inferences. Nevertheless, there appears to be a strong and significant association between childhood adversity and bipolar disorder.

We found some variances in this association when specific types of exposure were analysed separately. Emotional abuse was four times more likely to have occurred in bipolar disorder groups than in healthy controls, an effect seemingly larger than for other types of adversity. This is in contrast to a recent meta-analysis that observed roughly equivalent effect sizes for adversity subtypes on psychosis. ${ }^{15}$ Interestingly, parental loss did not significantly differ between bipolar and non-clinical samples. One explanation is that the impact of losing a parent is highly dependent on the context and stage at which it occurs. ${ }^{5}$ Indeed, research has suggested that younger age at parental loss, maternal loss in particular and death by unnatural causes are more strongly associated with a bipolar disorder diagnosis. ${ }^{2,50,51}$ Refuting our initial hypothesis, the effect of childhood adversity on type 2 bipolar disorder, compared with type 1 disorder, did not reach statistical significance. Although the analysis included only four studies, it is possible that childhood adversity is associated with both the more severe and attenuated bipolar profiles.

Rates of childhood adversity were significantly greater in bipolar disorder when compared with major depression. However, this effect became non-significant when controlling for hypothetically missing studies. The absence of a stronger effect may have been due to the overrepresentation of studies considering parental loss, which did not appear to be elevated in bipolar disorder more generally. When repeating the analysis without effects pertaining to parental loss, individuals with bipolar disorder presented with higher levels of adversity compared with the major depression group. Nevertheless, it is difficult to draw firm conclusions concerning the specificity of childhood adversity in the two disorders.

The results showed no significant difference in the rates of childhood adversity between individuals diagnosed with bipolar disorder and those with schizophrenia. A wealth of research has focused on the role of childhood adversity in the development of psychosis; ${ }^{15}$ our findings suggest similar levels of adversity in bipolar disorder. Interestingly, correlational studies have showed associations between childhood adversity and psychotic experiences in bipolar disorder. ${ }^{8}$ Future research should explore the exact pathways by which specific forms of adversity lead to particular symptom clusters.

The analysis revealed high levels of statistical heterogeneity, which allows for less confidence in the estimated effect sizes, but is not surprising given the methodological and analytic variances in the identified studies. For example, measures of childhood adversity included national registers, questionnaires, survey items and semi-structured interviews. Furthermore, studies differed in terms of diagnostic assessments (e.g. the Structured Clinical Interview for DSM Disorders, the Composite International Diagnostic Interview) and inclusion criteria (e.g. adolescents, adults), with two studies restricting their analysis to type 1 bipolar disorder. ${ }^{26,42}$ Although the analyses allowed for the examination of some potential sources of heterogeneity (e.g. the impact of study design), the limited number of identified studies prevented the authors from testing the impact of other methodological differences on effect sizes. In the presence of further publications, future reviews might wish to explore whether such methodological and clinical variations moderate the association between childhood adversity and bipolar disorder.

\section{Study limitations}

There are some limitations of this meta-analysis and of the research literature more generally. Recall bias and illness representations may confound retrospective reporting of childhood adversity. ${ }^{17}$ In the absence of long-term prospective research it is impossible to reach a definitive conclusion on the causal link between childhood adversity and bipolar disorder. It is feasible that, in some cases, early or prodromal symptoms in childhood might place greater strain on parenting, which could contribute to dysfunctional relationships. Therefore, a genetic predisposition to bipolar disorder might increase levels of childhood adversity. Similarly, we note the absence of studies carefully examining graded (i.e. dose-response) relationships, which in conjunction with the investigation of putative biological and psychosocial mechanisms might enable the identification of plausible pathogenic pathways linking adversity to bipolar psychopathology. Last, the adversity subtypes were not statistically independent, making it difficult to draw firm conclusions on the specificity of adversity subtypes on bipolar disorder.

\section{Clinical implications}

Given the association between childhood adversity and bipolar disorder, practitioners should carefully enquire about their clients' past adverse experiences, including emotional abuse. Read et al have provided guidance on how clinicians might conduct these conversations and respond sensitively to and deal with disclosures. ${ }^{52}$ Identification of childhood adversity should then lead to its integration into personalised formulations of clients' difficulties and the provision of appropriate support and interventions.

In conclusion, childhood adversity appears to be associated with the development of bipolar disorder. Rates of childhood adversity in bipolar disorder appear to be similar to those observed in psychosis and major depression. Researchers should explore the ways in which childhood adversity interacts with cognitive, behavioural and biological factors. They should also investigate the potential impact of alternative forms of adversity such as bullying and witnessing domestic violence. Further prospective research exploring dose-response and accounting for genetic effects would help to elucidate the nature of the relationship between childhood adversity and bipolar symptoms. The findings have implications for the study and treatment of bipolar disorder.

J. E. Palmier-Claus, PhD, ClinPsyD, Psychosis Research Unit, Greater Manchester West National Health Service Foundation Trust, Manchester, and Institute for Brain, Behaviour and Mental Health, University of Manchester; K. Berry, PhD, ClinPsyD, S. Bucci, DClinPsy, w. Mansell, DClinPsy, F. Varese, PhD, ClinPsyD, Section for Clinical and Health Psychology, University of Manchester, Manchester, UK

Correspondence: Dr J. E. Palmier-Claus, Psychosis Research Unit, Greater Manchester West NHS Foundation Trust, Harrop House, Bury New Road Prestwich M25 3BL, UK. Email: Jasper.Palmier-Claus@manchester.ac.uk

First received 8 Dec 2015, final revision 26 Mar 2016, accepted 25 Jun 2016

\section{Acknowledgements}

The authors thank Louise Laverty and Rebecca Harrop for their assistance with reliability checking; and Alex Fowke, Daniela Caldirola, Delfina Janiri, Gianfranco Spalletta, Jonathan Savitz, Lauren Alloy, Lize van der Merwe, Luciano de Souza, Monica Aas, Netta Horesh, Po-Hsiu Kuo, Stuart Watson and Toshi Furukawa for the information they supplied.

\section{References}

1 Sanchez-Moreno J, Martinez-Aran A, Tabares-Seisdedos R, Torrent C, Vieta E, Ayuso-Mateos J. Functioning and disability in bipolar disorder: an extensive review. Psychother Psychosom 2009; 78: 285-97.

2 Michalak EE, Yatham LN, Lam RW. Quality of life in bipolar disorder: a review of the literature. Health Qual Life Outcomes 2005; 3: 72.

3 Harris EC, Barraclough B. Suicide as an outcome for mental disorders. A meta-analysis. Br J Psychiatry 1997; 170: 205-28. 
4 Simhandl C, Konig B, Amann BL. A prospective 4-year naturalistic follow-up of treatment and outcome of 300 bipolar I and II patients. J Clin Psychiatry 2014; 75: 254-63.

5 Etain B, Henry C, Bellivier F, Mathieu F, Leboyer M. Beyond genetics: childhood affective trauma in bipolar disorder. Bipolar Disord 2008; 10 867-76.

6 Arnow BA. Relationships between childhood maltreatment, adult health and psychiatric outcomes, and medical utilization. J Clin Psychiatry 2004; 65 $10-5$

7 Etain B, Aas M, Andreassen OA, Lorentzen S, Dieset I, Gard S, et al Childhood trauma is associated with severe clinical characteristics of bipolar disorders. J Clin Psychiatry 2013; 74: 991-8.

8 Upthegrove R, Chard C, Jones L, Gordon-Smith K, Forty L, Jones I, et al. Adverse childhood events and psychosis in bipolar affective disorder. Br J Psychiatry 2015; 206: 191-7.

9 Daruy-Filho L, Brietzke E, Lafer B, Grassi-Oliveira R. Childhood maltreatment and clinical outcomes of bipolar disorder. Acta Psychiatr Scand 2011; 124: 427-34.

10 Maniglio R. Prevalence of child sexual abuse among adults and youths with bipolar disorder: a systematic review. Clin Psychol Rev 2013; 33 561-73

11 Maniglio R. The impact of child sexual abuse on the course of bipolar disorder: a systematic review. Bipolar Disord 2013; 15: 341-58.

12 Tsuchiya KJ, Byrne M, Mortensen PB. Risk factors in relation to an emergence of bipolar disorder: a systematic review. Bipolar Disord 2003, 5: $231-42$.

13 Alloy LB, Abramson LY, Smith JM, Gibb BE, Neeren AM. Role of parenting and maltreatment histories in unipolar and bipolar mood disorders: mediation by cognitive vulnerability to depression. Clin Child Fam Psych 2006; 9: 23-64.

14 Alloy LB, Abramson LY, Urosevic S, Walshaw PD, Nusslock R, Neeren AM. The psychosocial context of bipolar disorder: environmental, cognitive, and developmental risk factors. Clin Psychol Rev 2005; 25: 1043-75.

15 varese F, Smeets F, Drukker M, Lieverse R, Lataster T, viechtbauer W, et al. Childhood adversities increase the risk of psychosis: a meta-analysis of patient-control, prospective- and cross-sectional cohort studies. Schizophr Bull 2012; 38: 661-71.

16 Rubino IA, Nanni RC, Pozzi DM, Siracusano A. Early adverse experiences in schizophrenia and unipolar depression. J Ment Nerv Dis 2009; 197: 65-8.

17 Fisher HL, Hosang GM. Childhood maltreatment and bipolar disorder: critical review of the evidence. Mind Brain J Psychiatry 2010; 1: 75-85.

18 Scott KM, Smith DR, Ellis PM. Prospectively ascertained child maltreatment and its association with DSM-IV mental disorders in young adults. Arch Gen Psychiatry 2010; 67: 712-9.

19 Wells G, Shea B, O'Connell D, Peterson J, Welch V, Losos M, et al. The Newcastle-Ottawa Scale (NOS) for Assessing the Quality of Nonrandomised Studies in Meta-analyses. 2000 (http://www.ohri.ca/ programs/clinical_epidemiology/oxford.asp).

20 Tolin DF, Foa EB. Sex differences in trauma and posttraumatic stress disorder: a quantitative review of 25 years of research. Psychol Bull 2006; 132: 959-92.

21 Stikkelbroek $Y$, Prinzie $P$, de Graaf R, ten Have M, Cuijpers P. Parental death during childhood and psychopathology in adulthood. Psychiatry Res 2012; 198: $516-20$.

22 Laursen TM, Munk-Olsen T, Nordentoft M, Bo MP. A comparison of selected risk factors for unipolar depressive disorder, bipolar affective disorder, schizoaffective disorder, and schizophrenia from a Danish population-based cohort. J Clin Psychiat 2007; 68: 1673-81.

23 Fowke A, Ross S, Ashcroft K. Childhood maltreatment and internalized shame in adults with a diagnosis of bipolar disorder. Clin Psychol Psychother 2012; 19: 450-7

24 Afifi TO, MacMillan HL, Boyle M, Taillieu T, Cheung K, Sareen J. Child abuse and mental disorders in Canada. CMAJ 2014; 186: E324-32.

25 Molnar BE, Buka SL, Kessler RC. Child sexual abuse and subsequent psychopathology: results from the National Comorbidity Survey. Am J Public Health 2001; 91: 753-60.

26 Gilman SE, Ni M, Dunn EC, Breslau J, McLaughlin KA, Smoller JW, et al. Contributions of the social environment to first-onset and recurrent mania. Mol Psychiatry 2015; 20: 329-36.

27 Bernstein DP, Fink L. Childhood Trauma Questionnaire: A Retrospective Selfreport: Manual. Psychological Corporation, 1998.

28 Agid O, Shapira B, Zislin J, Ritsner M, Hanin B, Murad H, et al. Environment and vulnerability to major psychiatric illness: a case control study of early parental loss in major depression, bipolar disorder and schizophrenia. Mol Psychiatry 1999; 4: 163-72.

29 Furukawa T, Ogura A, Hirai T, Fujihara S, Kitamura T, Takahashi $\mathrm{K}$ Early parental separation experiences among patients with bipola disorder and major depression: a case-control study. J Affect Disord 1999; 52: 85-91.

30 Rucklidge JJ. Psychosocial functioning of adolescents with and without paediatric bipolar disorder. J Affect Disord 2006; 91: 181-8.

31 Grandin LD, Alloy LB, Abramson LY. Childhood stressful life events and bipolar spectrum disorders. J Soc Clin Psychol 2007; 26: 460-78.

32 Savitz JB, Van Der Merwe L, Stein DJ, Solms M, Ramesar RS. Neuropsychological task performance in bipolar spectrum illness: genetics, alcohol abuse, medication and childhood trauma. Bipolar Disord 2008; 10: 479-94.

33 Etain B, Mathieu F, Henry C, Raust A, Roy I, Germain A, et al. Preferential association between childhood emotional abuse and bipolar disorder. J Trauma Stress 2010; 23: 376-83.

34 Horesh N, Iancu I. A comparison of life events in patients with unipolar disorder or bipolar disorder and controls. Compr Psychiatry 2010; 51: 157-64.

35 Konradt CE, Jansen K, Magalhaes PVdS, Pinheiro RT, Kapczinski FP, da Silva RA, et al. Early trauma and mood disorders in youngsters. Rev Psiquiatr Clin 2013; 40: 93-6.

36 Aas M, Aminoff SR, Lagerberg TV, Etain B, Agartz I, Andreassen OA, et al. Affective lability in patients with bipolar disorders is associated with high levels of childhood trauma. Psychiatry Res 2014; 218: 252-5.

37 Chen YC, Kao CF, Lu MK, Yang YK, Liao SC, Jang FL, et al. The relationship of family characteristics and bipolar disorder using causal-pie models. Eur Psychiat 2014; 29: 36-43.

38 Watson S, Gallagher P, Dougall D, Porter R, Moncrieff J, Ferrier IN et al. Childhood trauma in bipolar disorder. Aust NZ J Psychiatry 2014; 48: $564-70$.

39 Janiri D, Sani G, Danese E, Simonetti A, Ambrosi E, Angeletti G, et al. Childhood traumatic experiences of patients with bipolar disorder type I and type II. J Affect Disord 2015; 175: 92-7.

40 Alnæs R, Torgersen S. Mood disorders: developmental and precipitating events. Can J Psychiatry 1993; 38: 217-24.

41 Darves-Bornoz JM, Lemperiere T, Degiovanni A, Gaillard P. Sexual victimization in women with schizophrenia and bipolar disorder. Soc Psychiatry Psychiatr Epidemiol 1995; 30: 78-84.

42 Hlastala SA, McClellan J. Phenomenology and diagnostic stability of youths with atypical psychotic symptoms. J Child Adolesc Psychopharmacol 2005; 15: 497-509.

43 Hyun M, Friedman SD, Dunner DL. Relationship of childhood physical and sexual abuse to adult bipolar disorder. Bipolar Disord 2000; 2: 131-5.

44 Watson S, Owen BM, Gallagher P, Hearn AJ, Young AH, Ferrier IN. Family history, early adversity and the hypothalamic-pituitary-adrenal (HPA) axis: mediation of the vulnerability to mood disorders. Neuropsychiat Dis Treat 2007; 3: 647-53.

45 Angst J, Gamma A, Rossler W, Ajdacic V, Klein DN. Childhood adversity and chronicity of mood disorders. Eur Arch Psychiatry Clin Neurosci 2011; 261: 21-7.

46 Alvarez MJ, Roura P, Oses A, Foguet Q, Sola J, Arrufat FX. Prevalence and clinical impact of childhood trauma in patients with severe mental disorders. J Nerv Ment Dis 2011; 199: 156-61.

47 Parker G, Fletcher K, Mccraw S, Futeran S, Hong M. Identifying antecedent and illness course variables differentiating bipolar I, bipolar II and unipolar disorders. J Affect Disord 2013; 148: 202-9.

48 Perna G, Vanni G, Di Chiaro NV, Cavedini P, Caldirola D. Childhood trauma, temperament, and character in subjects with major depressive disorder and bipolar disorder. J Nerv Ment Dis 2014; 202: 695-8.

49 Neeren AM, Alloy LB, Abramson LY. History of parenting and bipolar spectrum disorders. J Soc Clin Psychol 2008; 27: 1021-44.

50 Mortensen PB, Pedersen C, Melbye M, Mors O, Ewald H. Individual and familial risk factors for bipolar affective disorders in Denmark. Arch Gen Psychiatry 2003; 60: 1209-15.

51 Nickerson A, Bryant RA, Aderka IM, Hinton DE, Hofmann SG. The impacts of parental loss and adverse parenting on mental health: findings from the National Comorbidity Survey-Replication. Psychol Trauma 2013; 5: 119-27.

52 Read J, Hammersley P, Rudegeair T. Why, when and how to ask about childhood abuse. Adv Psychiatr Treat 2007; 13: 101-10. 